\title{
3D Jet Tomography of the Twisted Color Glass Condensate
}

\author{
A. Adil ${ }^{*}$ M. Gyulassy \\ Columbia University, Department of Physics, 538 West 120-th Street, New York, NY 10027
}

(Dated: September 15, 2021)

\begin{abstract}
Jet Tomography is proposed as a new test of Color Glass Condensate (CGC) initial conditions in non-central $A+A$ collisions. The $k_{T}$ factorized CGC formalism is used to calculate the rapidity twist in the reaction plane of both the bulk low $p_{T}<2 \mathrm{GeV}$ matter as well as the rare high $p_{T}>6$ $\mathrm{GeV}$ partons. Unlike conventional perturbative QCD, the initial high $p_{T}$ CGC gluons are shown to be twisted even further away from the beam axis than the the low $p_{T}$ bulk at high rapidities $|\eta|>2$. Differential directed flow $v_{1}\left(p_{T}>6,|\eta|>2\right)$ is proposed to test this novel high $p_{T}$ rapidity twist predicted by the CGC model.
\end{abstract}

PACS numbers: 12.38.Mh; 24.85.+p; 25.75.-q

Introduction: It was pointed out in Ref. [1] that the QCD matter produced in high energy noncentral $A+A$ nuclear reactions violates (locally) Bjorken longitudinal boost invariance in the transverse plane even if the global rapidity distribution, $d N / d \eta$, is independent of $\eta=$ $\sinh ^{-1}\left(p_{z} / m_{\perp}\right)$. The intrinsic longitudinal boost noninvariance occurs even in symmetric $A+A$ reations because locally in the transverse $\mathbf{x}_{\perp}$ plane, the initial gluon density, $\rho_{g}\left(\eta, \mathbf{x}_{\perp}\right)=d N_{g} / d \eta d^{2} \mathbf{x}_{\perp}$, has a generic "trapezoidal" form in the rapidity variable. This peculiar structure arises in noncentral $(b>0)$ collisions because there is an asymmetry between the local number of interacting projectile and target nucleons, $\Delta \nu\left(\mathbf{x}_{\perp} ; b>0\right) \sim A^{1 / 3}$, that can vary by an order of magnitude in heavy nuclei with the transverse coordinate, $\mathbf{x}_{\perp}$. Since low $p_{T}$ bulk matter is known to be produced proportional to the number of participating nucleons, the slope of the rapidity trapezoid varies with $\mathbf{x}_{\perp}$ as $d \rho / d \eta \propto \Delta \nu\left(\mathbf{x}_{\perp} ; b\right) / 2 Y \sim$ $A^{1 / 3} / \log s$, where $2 Y=\log s$ is the rapidity gap between the projectile and target nuclei. At infinite energies this slope approaches zero, but at the Relativistic Heavy Ion Collider (RHIC) and the Large Hadron Collider (LHC) it may be large enough to be observable via $3 \mathrm{D}\left(p_{\perp}, \phi, \eta\right)$ extensions [1] of jet tomography [2].

The trapezoidal rapidity asymmetry has been observed at all energies in $p+A$ reactions and it was also clearly seen in $D+A u$ reactions at RHIC [3, 4]. The data are well reproduced by phenomenological soft + hard (string+mini-jet) models such as [5, [6, 7]. However, these trapezoidal features are also well reproduced by gluon saturation models such as in the Kharzeev-Levin-Nardi (KLN) 8] implementation of the Color Glass Condenstate (CGC) theory 9, 10] of low x parton initial conditions.

However, the phenomenological soft+hard and CGC approaches differ significantly in their predictions for moderately high $p_{T}$ partons above $Q_{s}$. In the former case, the high $p_{T}$ parton production is calculated from the collinear factorized on shell $g g \rightarrow g g$ approximation of $\mathrm{pQCD}$ for hard processes. In the KLN/CGC approach both soft and hard partons are calculated using the $k_{T}$ factorized off-shell $g g \rightarrow g$ gluon fusion approximation to QCD parton production [11]. The advantage of collinear factorized approximations is that they employ experimentally well determined nucleon parton structure functions, $x G_{N}\left(x, Q^{2}\right)$. In addition, corrections beyond lowest order can be systematically evaluated. The main disadvantage in applications to $A+A$ is that possible nonlinear nuclear effects at low x could significantly modify the assumed linear relation $G_{A}=A G_{N}$ in different kinematic regimes.

In contrast, KLN/CGC predictions are based on the convolution of unintegrated nuclear gluon distributions, $\phi_{A}\left(x, k_{T}^{2}\right)$ via the Gribov-Levin-Ryskin (GLR) formula. This approach has the advantage of including a nonlinear gluon evolution as $x \rightarrow 0$ via a single gluon saturation scale $Q_{s}(x, A)$ that can be determined in the weak coupling but strong field approximation. The main disadvantage of this approach is the uncertainty related to how to take the $A \rightarrow 1$ nucleon limit, $\phi_{N}\left(x, k_{T}^{2}\right)$. This limit is important phenomenologically because nuclear modification is measured relative to the $N+N$ baseline. In addition, near the nuclear surface the participant nucleon density decreases rapidly and $Q_{s}$ is driven below $1 \mathrm{GeV}$ where the weak coupling strong field approximations become suspect. Since the surface regions in finite $\mathrm{A}+\mathrm{A}$ contribute significantly $(\sim 10-20 \%)$ to the global $d N / d y$, the specific implementation of the low $A$ limit is important to devise experimental tests sensitive to this limit of CGC. Unlike the integrated parton distributions, there is no general consensus yet on the form of $\phi_{N}$ [12]. Additional theoretical uncertainty is associated with the unknown applicability range of the first 
order GLR $k_{T}$ factorization formulation [11] as compared to proposed higher order nonlinear generalizations 13.

At RHIC the strongest support for the KLN/CGC approach is its remarkable ability to reproduce the extensive systematics of the energy and nuclear size dependence of the global $p_{T}$ integrated $d N_{c h} / d y$. This results from a specific dependence of the saturation scale, $Q_{s}$, on $\sqrt{s}$ and $A$. In contrast, phenomenological soft-hard models such as HIJING fail to reproduce the systematics because the separation scale, $p_{0} \sim 2 \mathrm{GeV}$, between soft and hard parton production was assumed to be independent of those variables. It is an important open question of how high in $p_{T}$ can the $k_{T}$-factorized KLN/CGC approximation be pushed in specifying the $A+A$ initial conditions versus how low in $p_{T}$ can the conventional collinear factorized approximation be pushed. Both experimental and theoretical control over the initial conditions in $A+A$ at RHIC are essential to strengthen the current case for the discovery of new forms of matter, the strongly coupled Quark Gluon Plasma (sQGP) and CGC, at RHIC [14, 15, 16].

In this letter a new jet tomographic approach is proposed based on extending the discussion in Ref. 1$]$ to KLN/CGC initial conditions of the sQGP bulk as well its extrapolation to high $p_{T} \gg Q_{s}$ jet partons. The idea is to exploit the difference between the geometric distributions of jets relative to that of the bulk matter as illustrated in Fig 1 We focus on the predicted azimuthal dependence of the jet quenching pattern, $R_{A A}\left(\eta, \phi, p_{\perp} ; b>0\right)$, and long range rapidity correlations induced by the generic intrinsic rapidity twist of the bulk matter.

At midrapidity, $\eta=0$, the elliptic asymmetry of the reaction geometry in noncentral $(b>0)$ in $A+A$ reactions leads to a well known elliptic asymmetry in jet quenching. However, the rapidity twist has no observable effect at mid-rapidity. At positive rapdities $\eta>2$, on the other hand the rapidity twist of the bulk shifts the center of mass away from $x=0$, while at negative rapidities that shift has opposite sign. In conventional pQCD, collinear factorized $g g \rightarrow g g$ predicts a jet distribution that is proportional to the local binary collision density, $\sigma_{N N} T_{B}\left(r_{+}\right) T_{A}\left(r_{-}\right)$, where $r_{ \pm}=\sqrt{\left(x \pm \frac{b}{2}\right)^{2}+y^{2}}$, and $T_{A}(\mathbf{x})$ is the Glauber nuclear profile function [1]. The jets are therefore produced symmetrically about $\mathbf{x}_{\perp}=0$ at all $\eta$. The collinear factorized jet density therefore has no rapidity twist as illustrated by the grey ellipse in Fig 1

The bulk matter density is effectively rotated away from the beam axis because the bulk density varies approximately as $\left\{(Y-\eta) T_{A}\left(r_{+}\right)+(Y+\eta) T_{A}\left(r_{-}\right)\right\} / 2 Y$, which is not reflection symmetric about $\mathbf{x}_{\perp}=0$ away from midrapidity. At $p_{T}<Q_{s}$ the CGC model produces approximately the same rapidity twist as wounded nucleon string models since this is a direct consequence of local participant versus binary collision scaling of the bulk. However, due to the nonlinear equation determin-

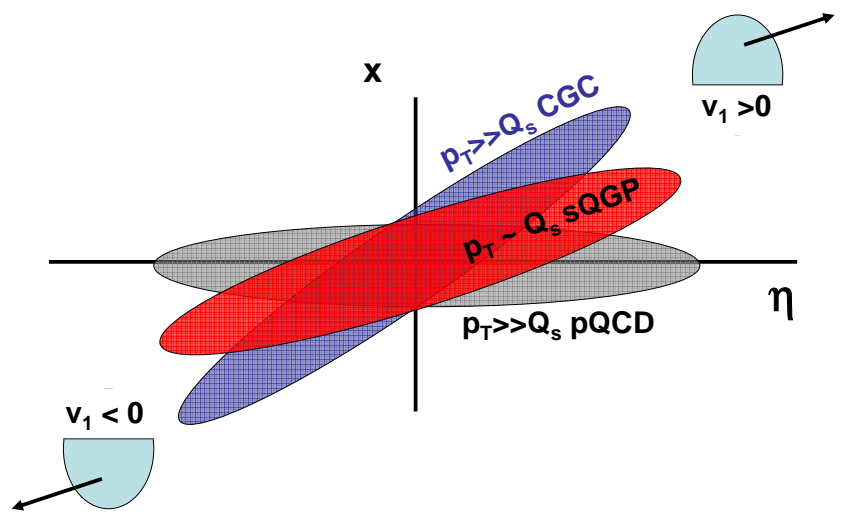

FIG. 1: (Color Online) Illustration of the initially twisted sQGP gluon density [1] relative to the beam axis in the $(x, \eta)$ reaction plane. CGC [8], BGK [5], and HIJING 7] predict this generic low $p_{T} \lesssim Q_{s}$ locally boost non-invariant structure. Also shown are the relative rotations of the high $p_{T} \gg Q_{s}$ jet partons in the $k_{T}$ factorized CGC model as well as conventional collinear factored $\mathrm{pQCD}$. Jet quenching through the sQGP leads to opposite sign first azimuthal harmonic moment, $v_{1}\left(p_{T} \gg Q_{s}, \eta\right)$ in the two approaches. The projectile and spectator nuclei are indicated by half circles together with the sign convention of low $p_{T}$ directed flow $v_{1}$.

ing the local saturation scale $Q_{s}\left(\mathbf{x}_{\perp}, x\right)$ the bulk density surface region can sharpen significantly over conventional Wood-Saxon geometry included in $T_{A}\left(\mathbf{x}_{\perp}\right)$. This surprising change of the bulk surface geometry in the KLN implementation of CGC was first pointed out by Hirano and Nara 18.

However, as we show below, at higher $p_{T}$ the CGC predicts even greater rapdity twist away from the beam axis than the bulk as shown below and illustrated in Fig 1 In this paper, we explore some tomographic consequences of using the $k_{T}$ factorized formalism and CGC type unintegrated gluon distributions to produce both the bulk and jet matter in the reaction. This anomalous rapidity twist effect is opposite to that discussed in [1]. This effect occurs because the different nuclei are probed at asymmetric Bjorken momentum fractions while producing high $p_{T}$ matter.

The Local Gluon Distribution: The local generalization of $k_{T}$-factorization GLR formula 11] used by KLN [8] and Hirano and Nara [18] is given by

$$
\begin{aligned}
& \frac{d N_{g}}{d p_{T} d^{2} x_{T} d \eta}=\frac{4 \pi}{C_{F}} \frac{\alpha_{s}\left(p_{T}^{2}\right)}{p_{T}} \int^{p_{T}} d^{2} k_{T} \times \\
& \phi_{A}\left(x_{1},\left(\frac{\vec{k}_{T}+\vec{p}_{T}}{2}\right)^{2} ; \vec{x}_{T}\right) \phi_{B}\left(x_{2},\left(\frac{\vec{k}_{T}-\vec{p}_{T}}{2}\right)^{2} ; \vec{x}_{T}\right) .
\end{aligned}
$$

$C_{F}=\frac{N_{C}^{2}-1}{2 N_{C}}$ and the collinear momentum fractions are given by kinematics, $x_{1,2}=p_{T} \exp ( \pm \eta) / \sqrt{s}$. The QCD coupling, $\alpha_{s}$, is evaluated at $p_{T}^{2}$ and regulated at low $p_{T}$ by imposing a maximum value $\alpha_{\max }=0.5$. Note that we use $\eta$ to denote the rapidity rather than the pseudo 
rapidity as in [1].

$\phi_{A, B}$ are the unintegrated gluon distributions which, in principle, possess a Bjorken $x$ dependence determined by nonlinear evolution equations of the CGC theory 9,10 , and their $k_{T}$ dependence is fixed by a characteristic saturation momentum, $Q_{s}(x)$. In the McLerran-Venugopalan approach 19] the gluon distribution is suppressed below the saturation scale $\phi_{A} \sim \log \left(Q_{S}^{2} / k_{T}^{2}\right)$ compared to the perturbative form $\phi_{A} \sim k_{T}^{-2}$. We use a parameterization similar to the KLN model approach as used in [18]. However, we use (for numerical convenience) the following Lorentzian form of $\phi_{A, B}$ for all values of $k_{T}$.

$$
\phi_{A}\left(x, \vec{k}_{T} ; \vec{x}_{T}\right)=\frac{\kappa}{\alpha_{s}\left(Q_{s, A}^{2}\right)} \frac{Q_{s, A}^{2}}{k_{T}^{2}+Q_{s, A}^{2}+\Lambda^{2}} .
$$

The momentum scale $\Lambda=0.2 \mathrm{GeV}$ is a regulator for the high rapidity $y>4.5$ region as in [18]. The constant $\kappa \sim 0.5$ is a parameter set to reproduce $d N_{g} / d \eta \sim 1000$ at midrapidity central collisions. The transverse coordinate dependence is implicit in the saturation momentum determined numerically for each nucleus.

$$
Q_{s, A}^{2}\left(x, \vec{x}_{T}\right)=\frac{2 \pi^{2}}{C_{F}} \alpha_{s}\left(Q_{s, A}^{2}\right) x G_{\mathrm{nuc}}\left(x, Q_{s, A}^{2}\right) T_{A}\left(\vec{x}_{T}\right),
$$

where $T_{A}$ is the Glauber profile of nucleus $A$. We use standard diffuse Woods-Saxon profiles. The projectile and target nucleii are set up such that the spectator $v_{1}$ is positive at forward rapidity.

The KLN parametrization is used for the nucleonic gluon distribution.

$$
x G_{\mathrm{nuc}}\left(x, Q^{2}\right)=K \log \left(\frac{Q^{2}+\Lambda^{2}}{\Lambda_{Q C D}^{2}}\right) x^{-\lambda}(1-x)^{n}
$$

The momentum scales $\Lambda$ and $\Lambda_{\mathrm{QCD}}$ are set to $0.2 \mathrm{GeV}$. The $x^{-\lambda}$ term accounts for the rapid growth of small $\mathrm{x}$ gluons while the factor of $(1-x)^{n}$ was introduced in KLN to account qualitatively for the rapid depletion of gluons as $x \rightarrow 1$ outside the small $\mathrm{x}$ framework of the CGC model. As in the KLN approach, we set $\lambda=0.2$ and $n=4 . K \sim 1.35$ is used to set $\left\langle Q_{s}^{2}(x=0.01)\right\rangle \sim 2$ $\mathrm{GeV}^{2}$ for central collisions at midrapidity.

Once the distribution shown in Eq. प is evaluated, we are ready to investigate the transverse plane dependence (and hence the intrinsic spatial twist) of the produced gluons as a function of $p_{T}$ and $\eta$. We measure this shift of material away from the centre of the transverse reaction plane by calculating the average horizontal transverse coordinate.

$$
\langle x\rangle\left(p_{T}, \eta\right)=\frac{\int d^{2} x_{T} x d N_{g} / d p_{T} d^{2} x_{T} d \eta}{\int d^{2} x_{T} d N_{g} / d p_{T} d^{2} x_{T} d \eta}
$$

Hereafter, $x$ is the transverse coordinate in the direction of the reaction plane, $\vec{b}$. Positive $x$ points toward the projectile $\eta=Y$ spectator displacement . Fig. 2 shows

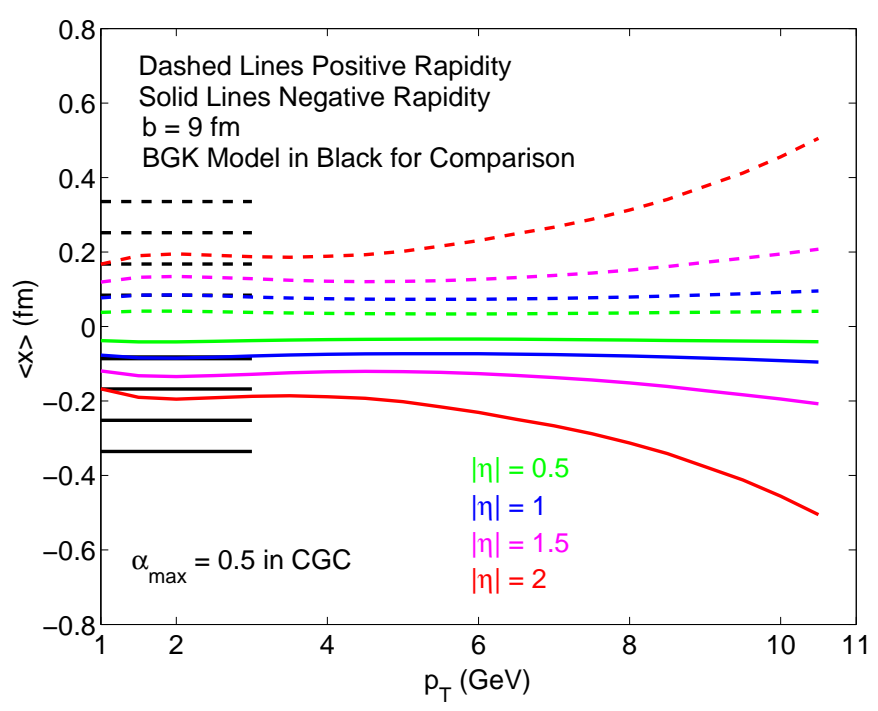

FIG. 2: (Color Online) The figure shows the average transverse spatial coordinate $\langle x\rangle$ for produced gluonic matter in the Brodksy-Gunion-Kuhn [5] type twist model as in [1], as well as in the current CGC model. Notice that, for BGK, there is no dependence on $p_{T}$ other than the assumed lack of twist for matter with $p_{T}>3 \mathrm{GeV}$. The CGC model, however, has a pronounced greater twist for higher $p_{T}>6 \mathrm{GeV}$.

$\langle x\rangle$ as a function of $p_{T}$ and $\eta$ for the hybrid BrodskyGunion-Kuhn (BGK) [5] participant and binary jet production model (as used in [1]) as well as for the current CGC model (for $b=9 \mathrm{fm}$ ). The rapidity twist of the BGK model is seen by the increasing $\langle x\rangle$ as $\eta$ increases for the bulk $p_{T} \leq 3 \mathrm{GeV}$ matter.

While the bulk $\langle x\rangle$ of the CGC model is similar to the bulk BGK shifts, the high $p_{T}$ shifts behave oppositely. In the two component BGK approach $\langle x\rangle=0$ for high $p_{T}$. We show in Fig. 2 that the rapidity twist $(d\langle x\rangle / d \eta)$ increases at high $p_{T}>6 \mathrm{GeV}$.

Tomography and the Inverse Twist: Jet tomographic analysis uses the attenuation of jet matter while passing through the bulk in order to gain information about the density profile of the bulk [2]. The observable used most commonly in tomographic analysis is the nuclear modification factor, $R_{A A}$, which measures the deviation of the produced nucleus-nucleus spectrum, if any, from a simple binary scaled p-p spectrum. The twist effect investigated in the previous section can be observed by looking at the $R_{A A}\left(p_{T}, \eta, \phi\right)$ of jets in the transverse plane.

The azimuthal dependence of $R_{A A}$ will change as a function of $\eta$ for a given $p_{T}$ jet due to the differing twist of the jet distribution over $\eta$. In order to calculate the $R_{A A}\left(p_{T}, \eta, \phi\right)$ we use the geometric model of Drees, Feng and Jia [20]. The nuclear modification factor is obtained 


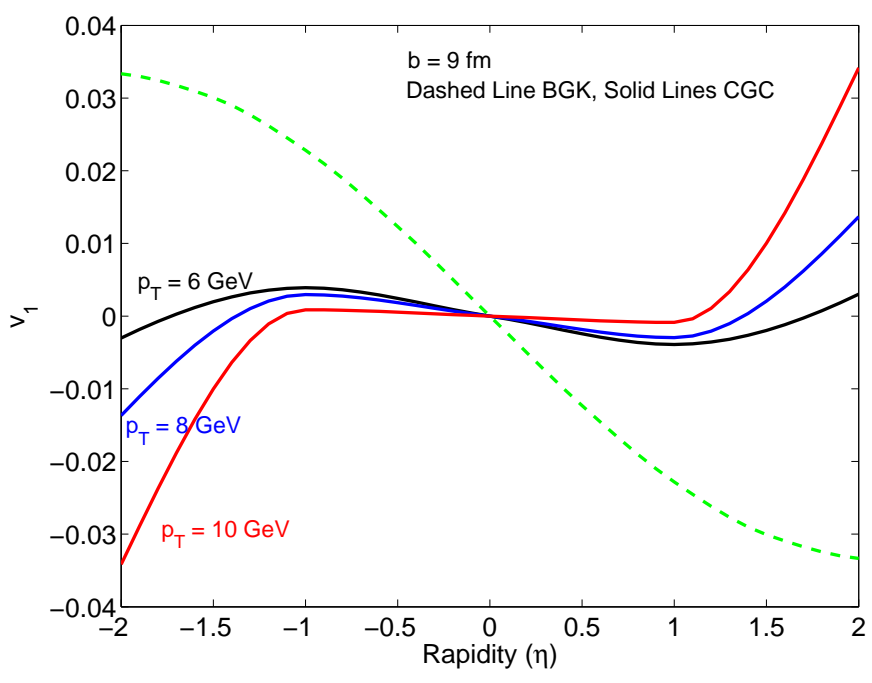

FIG. 3: (Color Online) The directed flow $v_{1}$ as a function of $\eta$ for different $p_{T}$ at $b=9 \mathrm{fm}$. Both the CGC model and BGK model are given for comparison.

by,

$$
R_{A A}\left(p_{T}, \eta, \phi\right)=\frac{\int d^{2} x_{T} e^{-\mu \chi\left(\vec{x}_{T}, \phi, \eta\right)} \frac{d N_{g}}{d p_{T} d^{2} x_{T} d \eta}\left(p_{T}, \eta\right)}{\int d^{2} x_{T} \frac{d N_{g}}{d p_{T} d^{2} x_{T} d \eta}\left(p_{T}, \eta\right)} .
$$

$\mu=0.04$ is the parameter used to set $R_{A A}(\eta=0, b=$ $0) \sim 0.25$. Opacity, $\chi$, is the line integral over the bulk distribution that is the cause of the attenuation experienced by the jet, calculated as in [20]. The length dependence of opacity is characteristic of radiative parton energy loss in Bjorken expanding matter.

We can use Eq. 6 to probe the twist. The anti-twist effect can most easily observed via the first azimuthal fourier moment of $R_{A A}\left(p_{T}, \eta, \phi\right)$, the directed flow $v_{1}$. Fig. 3 shows $v_{1}$ as a function of $\eta$ for different values of the $p_{T}$. Note that for all $p_{T}$ values there exists a rapidity at which the directed flow flips sign. This flip occurs at lower values of the rapidity for higher values of the $p_{T}$. The sign of $v_{1}$ is positive in CGC at high rapidity in the same direction as the projective spectator. The change in sign is a novel prediction using the KLN/CGC model. In conventional factorized QCD jet production, the high $p_{T} v_{1}$ is negative and in the same direction as the low $p_{T}$ bulk directed flow but increasing with $p_{T}$ as in hydrodynamics. Both models exhibit long range rapidity anticorrelations of the $v_{1}$. This anticorrelation may make it easier to measure the sign of $v_{1}$ and test up to how high $p_{T}$ can the KLN/CGC model describe initial conditions at RHIC.

Discussions with J. Harris, W. Horowitz, D. Kharzeev, L. McLerran, I. Vitev, X.N. Wang and Nu Xu are gratefully acknowledged. This work is supported in part by the United States Department of Energy under Grants No. DE-FG02-93ER40764.
* Electronic address: azfar@phys.columbia.edu

† Electronic address: gyulassy@phys.columbia.edu

‡ Electronic address: hirano@phys.columbia.edu

[1] A. Adil, M. Gyulassy, arXiv:nucl-th/0505044

[2] M. Gyulassy, I. Vitev, X. N. Wang and B. W. Zhang, arXiv:nucl-th/0302077 in "Quark Gluon Plasma 3", (editors: R.C. Hwa and X.N. Wang, World Scientific, Singapore.) p. 123-191; P. Jacobs and X. N. Wang, Prog. Part. Nucl. Phys. 54, 443 (2005) arXiv:hep-ph/0405125.

[3] B. B. Back et al. [PHOBOS Collaboration], Phys. Rev. Lett. 93, 082301 (2004) arXiv:nucl-ex/0311009.

[4] I. Arsene et al. [BRAHMS Collaboration], Phys. Rev. Lett. 94, 032301 (2005) arXiv:nucl-ex/0401025.

[5] S. J. Brodsky, J. F. Gunion and J. H. Kuhn, Phys. Rev. Lett. 39, 1120 (1977).

[6] B. Andersson, G. Gustafson and B. Nilsson-Almqvist, Nucl. Phys. B 281, 289 (1987).

[7] X. N. Wang and M. Gyulassy, multiple jet production in p p, p A and A A Phys. Rev. D 44, 3501 (1991); Comput. Phys. Commun. 83, 307 (1994) arXiv:nucl-th/9502021.

[8] D. Kharzeev, E. Levin and M. Nardi, Nucl. Phys. A 730, 448 (2004) [Erratum-ibid. A 743, 329 (2004)] arXiv:hep-ph/0212316; Nucl. Phys. A 747, 609 (2005) arXiv:hep-ph/0408050.

[9] E. Iancu, A. Leonidov and L. D. McLerran, Nucl. Phys. A 692, 583 (2001) arXiv:hep-ph/0011241.

[10] J. P. Blaizot and F. Gelis, Nucl. Phys. A 750, 148 (2005) arXiv:hep-ph/0405305.

[11] L. V. Gribov, E. M. Levin and M. G. Ryskin, Phys. Rept. 100, 1 (1983); Phys. Lett. B 100, 173 (1981); E Laenen and E. M. Levin, Ann. Rev. Nucl. Part. Sci. 44, 199 (1994).

[12] B. Andersson et al. [Small $\mathrm{x}$ Collaboration], Eur. Phys. J. C 25, 77 (2002) arXiv:hep-ph/0204115. A. Szczurek, Acta Phys. Polon. B 34, 3191 (2003) arXiv:hep-ph/0304129. L. Lonnblad and M. Sjodahl, JHEP 0505, 038 (2005) arXiv:hep-ph/0412111.

[13] N. N. Nikolaev and W. Schafer, Phys. Rev. D 71, 014023 (2005) arXiv:hep-ph/0411365.

[14] M. Gyulassy and L. McLerran, Nucl. Phys. A 750, 30 (2005) arXiv:nucl-th/0405013; See also articles by T.D.Lee, J.P. Blaizot, B. Muller, E. Shuryak, H. Stöcker, X.N. Wang, Nucl. Phys. A 750 (2005), pp.1-171.

[15] K. Adcox et al. [PHENIX Collab.], Nucl. Phys. A, 757 pp 184-283 (2005); B. B. Back et al. [PHOBOS Collab.], Nucl. Phys. A 757 pp 28-101 (2005); I. Arsene et al. [BRAHMS Collab.], Nucl. Phys. A 757 pp 1-27 (2005); J. Adams et al. [STAR Collab.], Nucl. Phys. A 757 pp 102-183 (2005);

[16] T. Hirano and M. Gyulassy, arXiv:nucl-th/0506049

[17] D. Kharzeev and M. Nardi, Phys. Lett. B 507, 121 (2001); D. Kharzeev and E. Levin, ibid. B, 523, 79 (2001); D. Kharzeev, E. Levin and M. Nardi, arXiv:hep-ph/0111315; D. Kharzeev, E. Levin and M. Nardi, Nucl. Phys. A, 730, 448 (2004).

[18] T. Hirano and Y. Nara, Nucl. Phys. A 743, 305 (2004) arXiv:nucl-th/0404039.

[19] L. D. McLerran and R. Venugopalan, Phys. Rev. D 49, 2233 (1994); 49, 3352 (1994); 50, 2225 (1994).

[20] A. Drees, H. Feng and J. Jia, arXiv:nucl-th/0310044 